\title{
Daratumumab proves effective in patients with newly diagnosed multiple myeloma
}

Bortezomib plus melphalan and prednisone is a widely used standard-of-care treatment for patients with newly diagnosed multiple myeloma who are ineligible for autologous stem-cell transplantation, and typically provides a progression-free survival (PFS) duration of 18-24 months. Now, findings of the phase III ALCYONE trial demonstrate the superiority of adding the anti-CD38 antibody daratumumab to this regimen.

A total of 706 patients with newly diagnosed multiple myeloma were randomly assigned to receive bortezomib plus melphalan and prednisone, with or without daratumumab. The overall response rate was $90.9 \%$ in the daratumumab group versus $73.9 \%$ in the control group, with complete responses observed in $42.6 \%$ and $24.4 \%$ of patients, respectively, and the 18-month PFS was $71.6 \%$ with daratumumab versus $50.2 \%$ with the control regimen $(P<0.001$ for all comparisons). Patients in the daratumumab group had similar risks of grade 3 or 4 neutropenia, thrombocytopenia and anaemia, albeit with daratumumab infusion-related reactions in $27.7 \%$ of patients.

These findings confirm the value of adding daratumumab to chemotherapy regimens for patients with multiple myeloma, and are consistent with the results of the VISTA and GIMEMA trials, which demonstrated the effectiveness of bortezomib and the ability to improve the tolerability of bortezomibcontaining regimens by reducing the dose of chemotherapy. Daratumumab is currently approved for use in patients with multiple myeloma after disease progression on at least one prior line of therapy; these observations might lead to the approval of daratumumab in the first-line setting. Long-term follow-up data are eagerly awaited.

Peter Sidaway

ORIGINAL ARTICLE Mateos, M.-V. et al. Daratumumab plus bortezomib, melphalan, and prednisone for untreated myeloma. N. Engl.J.Med. http://dx.doi.org/10.1056/NEJMoa1714678 (2017) 